\title{
Use of Computed Tomography and Positron Emission Tomography/Computed Tomography for Staging of Local Extent in Patients With Malignant Pleural Mesothelioma
}

Frauenfelder, Thomas ; Kestenholz, Peter ; Hunziker, Roger ; Nguyen, Thi Dan Linh ; Fries, Martina ; Veit-Haibach, Patrick ; Husmann, Lars ; Stahel, Rolf ; Weder, Walter ; Opitz, Isabelle

\begin{abstract}
PURPOSE The objective of this study was to determine the diagnostic value of computed tomography (CT) and positron emission tomography (PET)/CT for staging of malignant pleural mesothelioma (MPM) in patients undergoing induction chemotherapy. METHODS Sixty-two patients (median age, 61 years; female: $\mathrm{n}=9$ ) with proven MPM underwent CT after induction chemotherapy. Of these, 28 underwent additional PET/CT. Extrapleural pneumonectomy was performed for pathological TNM staging. Clinical TNM stage was assessed by 3 independent readers. Relative and absolute underestimation and overestimation were compared with pathological tumor stage. Sensitivity, specificity, and accuracy for differentiation between stages T2 and T3 were assessed. Interobserver agreement between the readers was analyzed ( ). RESULTS Positron emission tomography/CT and CT underestimated T stage in up to $30 \%$ of the cases. Positron emission tomography/CT had a higher accuracy for tumor extent compared with CT (PET/CT: 0.92; CT: 0.84). The accuracy for nodal staging was higher for CT than for PET/CT (PET/CT: 0.78; CT: 0.87). Concerning International Mesothelioma Interest Group classification, PET/CT improved the accuracy of preoperative staging compared with $\mathrm{CT}$ (PET/CT: 0.91; CT: 0.82). Interobserver agreement was moderate for CT (0.48-0.62) and good for PET/CT (0.640.83 ) for $\mathrm{T}$ staging. For nodal staging, interobserver agreement was fair to moderate for $\mathrm{CT}$ and good for PET/CT (CT: 0.37-0.51; PET/CT: 0.73-0.76). CONCLUSIONS Positron emission tomography/CT is more accurate and has a lower interobserver variability for clinical intrathoracic staging of MPM compared with CT. Nevertheless PET/CT underestimated tumor stage in a substantial number of cases, showing the need for a more accurate imaging technology or approach.
\end{abstract}

DOI: https://doi.org/10.1097/RCT.0000000000000174

Posted at the Zurich Open Repository and Archive, University of Zurich

ZORA URL: https://doi.org/10.5167/uzh-100567

Journal Article

Published Version

Originally published at:

Frauenfelder, Thomas; Kestenholz, Peter; Hunziker, Roger; Nguyen, Thi Dan Linh; Fries, Martina; VeitHaibach, Patrick; Husmann, Lars; Stahel, Rolf; Weder, Walter; Opitz, Isabelle (2015). Use of Computed Tomography and Positron Emission Tomography/Computed Tomography for Staging of Local Extent in Patients With Malignant Pleural Mesothelioma. Journal of Computer Assisted Tomography, 39(2):160165.

DOI: https://doi.org/10.1097/RCT.0000000000000174 


\title{
Use of Computed Tomography and Positron Emission Tomography/Computed Tomography for Staging of Local Extent in Patients With Malignant Pleural Mesothelioma
}

\author{
Thomas Frauenfelder, MD, * Peter Kestenholz, MD, † Roger Hunziker, MD, * Thi Dan Linh Nguyen, MD, * \\ Martina Fries, $\uparrow$ Patrick Veit-Haibach, MD, * Lars Husmann, MD, $\neq$ Rolf Stahel, MD, $\S$ \\ Walter Weder, MD, $\dagger$ and Isabelle Opitz, MD $\dagger$
}

\begin{abstract}
Purpose: The objective of this study was to determine the diagnostic value of computed tomography $(\mathrm{CT})$ and positron emission tomography (PET)/CT for staging of malignant pleural mesothelioma (MPM) in patients undergoing induction chemotherapy.

Methods: Sixty-two patients (median age, 61 years; female: $n=9$ ) with proven MPM underwent CT after induction chemotherapy. Of these, 28 underwent additional PET/CT. Extrapleural pneumonectomy was performed for pathological TNM staging. Clinical TNM stage was assessed by 3 independent readers. Relative and absolute underestimation and overestimation were compared with pathological tumor stage. Sensitivity, specificity, and accuracy for differentiation between stages T2 and T3 were assessed. Interobserver agreement between the readers was analyzed ( $\kappa)$. Results: Positron emission tomography/CT and CT underestimated $\mathrm{T}$ stage in up to $30 \%$ of the cases. Positron emission tomography/CT had a higher accuracy for tumor extent compared with CT (PET/CT: 0.92; CT: 0.84). The accuracy for nodal staging was higher for CT than for PET/CT (PET/CT: 0.78; CT: 0.87). Concerning International Mesothelioma Interest Group classification, $\mathrm{PET} / \mathrm{CT}$ improved the accuracy of preoperative staging compared with CT (PET/CT: 0.91; CT: 0.82). Interobserver agreement was moderate for CT $(0.48-0.62)$ and good for $\mathrm{PET} / \mathrm{CT}(0.64-0.83)$ for $\mathrm{T}$ staging. For nodal staging, interobserver agreement was fair to moderate for CT and good for PET/CT (CT: $0.37-0.51$; PET/CT: 0.73-0.76).

Conclusions: Positron emission tomography/CT is more accurate and has a lower interobserver variability for clinical intrathoracic staging of MPM compared with CT. Nevertheless PET/CT underestimated tumor stage in a substantial number of cases, showing the need for a more accurate imaging technology or approach.
\end{abstract}

Key Words: malignant pleural mesothelioma, PET/CT, CT, tumor staging (J Comput Assist Tomogr 2014;00: 00-00)

omputed tomography (CT), magnetic resonance imaging (MRI), and positron emission tomography combined with CT (PET/CT) are the imaging modalities of choice for noninvasive staging. ${ }^{1-3}$ In particular, CT is widely used for clinical staging. Although imaging is essential to the diagnosis, assessment, and monitoring of malignant pleural mesothelioma (MPM), the complex morphology and growth pattern of MPM create unique challenges for imaging acquisition and interpretation. ${ }^{4}$ Each modality, because of their differences in scientific basis and technical capability, contributes to a better understanding of the genesis, biology, and biochemical response of MPM, allowing further development

From the *Institute of Diagnostic and Interventional Radiology, $\dagger$ Division of Thoracic Surgery, †Clinic of Nuclear Medicine, and §Clinic of Oncology, University Hospital of Zurich, Zurich, Switzerland.

Received for publication June 11, 2014; accepted September 25, 2014.

Reprints: Thomas Frauenfelder, MD, Institute of Diagnostic and Interventional

Radiology, University Hospital of Zurich, Rämistrasse 100, 8091 Zürich,

Switzerland (e-mail: Thomas.frauenfelder@usz.ch).

The authors declare no conflict of interest.

Copyright (C) 2014 by Lippincott Williams \& Wilkins of new treatment strategies. ${ }^{4}$ Whereas earlier studies have shown that CT and MRI failed to accurately define TNM tumor stage, the results of newer studies investigating the accuracy of CT, $\mathrm{PET} / \mathrm{CT}$, and MRI for staging of MPM are more promising. ${ }^{3,5-8}$

Clinical staging in patients with MPM is important for decision making in terms of patient selection for multimodality treatment, which currently offers the best survival. ${ }^{9}$ It is still unclear which patients will benefit from either a systemic or surgical treatment, as the management of this uncommon cancer is based on small data sets from single-center, retrospective studies. ${ }^{10-12}$ To determine treatment and prognosis and to make studies comparable, a correct and uniform staging system is very important. At least 5 systems for MPM are known in the literature. ${ }^{13}$ Early staging systems, like those from Butchard or Sugarbaker, do not use the T, N, and M descriptors as applied in the classic TNM classification of tumors. ${ }^{12-14}$ In 1995, the International Mesothelioma Interest Group (IMIG) introduced a new staging system based on the TNM classification in accordance with previous staging systems. ${ }^{15}$ This classification is similar to those for other solid tumors. As shown by Rusch et $\mathrm{al}^{6}$ in the initial analysis of the International Association for the Study of Lung Cancer (IASLC)/ IMIG database, there are concerns about the validity of the current staging system as the grouping into classes for both $\mathrm{T}$ stage and $\mathrm{N}$ stage does not reflect the tumor biology and behavior regarding the survival among different classes.

The objective of our study was to determine the diagnostic value of CT and PET/CT for staging of patients with pleural mesothelioma after induction chemotherapy. All patients had subsequent surgery, which was used as the criterion standard for the verification of imaging findings.

\section{MATERIALS AND METHODS}

A patient cohort of 62 patients undergoing induction chemotherapy followed by extrapleural pneumonectomy (EPP) over a 12 -year period was identified and retrospectively analyzed. All patients underwent CT after induction chemotherapy and before EPP, which served as the standard of reference. None of these patients had distant metastases. Median interval between CT scan and EPP was 16 days (0-28 days). Fifty-five percent $(\mathrm{n}=34)$ had previous talc pleurodesis, and $85 \%(n=53)$ underwent mediastinoscopy prior to chemotherapy.

Contrast-enhanced CT scan was performed at a median of 16 days prior to EPP (0-28 days). Twenty-six of these 62 patients (median age, 62 years [range, 38-69 years]; female: $n=4$; male: $\mathrm{n}=22$ ) had additional PET/CT scan prior to EPP. Median interval between PET/CT scan and EPP was 17 days (1-41 days). Institutional review board approval for data collection and analysis was obtained. Informed consent was obtained from all patients.

The chest CT protocol consisted of a venous phase CT of the chest using a scan delay of 100 seconds after starting contrast agent application. This protocol was chosen based on the results of a prior study. ${ }^{17}$ Computed tomography was performed with 
TABLE 1. Distribution of Pathological T, N, and IMIG Stages Among Population

\begin{tabular}{lcccccccccccccc}
\hline $\begin{array}{l}\text { No. cases } \\
\text { per TNM Stage }\end{array}$ & Stage & & T1 & T2 & T3 & T4 & N0 & N1 & N2 & N3 & IMIG I & IMIG II & IMIG III & IMIG IV \\
\hline & CT group & $(\mathrm{n}=62)$ & 5 & 4 & 43 & 10 & 38 & 6 & 17 & 1 & 5 & 3 & 44 & 10 \\
& PET/CT group & $(\mathrm{n}=26)$ & 1 & 1 & 19 & 5 & 13 & 4 & 8 & 1 & 1 & 1 & 21 & 3 \\
\hline
\end{tabular}

either a 64-section or 256-section CT scanner (Siemens Sensation and Siemens Sensation Flash, Erlangen, Germany). Chest images were reconstructed using a sharp-edged tissue convolution kernel (B60f) and a medium-smooth soft tissue convolution kernel (B30f) at a slice thickness of $2 \mathrm{~mm}$ and increment of $1.7 \mathrm{~mm}$.

All patients were examined on 1 of 2 combined PET/CT inline systems (Discovery STE or Discovery RX; GE Healthcare, Milwaukee, Wis). First, a low-dose CT scan was acquired from the head to the pelvic floor using the following parameters: $140 \mathrm{kV}, 40 \mathrm{mAs}, 0.5 \mathrm{~s} /$ tube rotation, slice thickness of $4.25 \mathrm{~mm}$. Immediately after the CT acquisition, the PET emission scan was acquired covering the same scan range with either 180 seconds (Discovery STE, 3D mode) or 120 seconds (Discovery RX, 3D mode) emission time per cradle position with 7-slice overlap (matrix, $128 \times 128$ ). A total of 7 to 9 cradle positions were scanned, resulting in a total PET acquisition time of 14 to $21 \mathrm{~min}$. The CT data used for the attenuation correction and the images were reconstructed using a standard iterative algorithm (ordered subset expectation maximization). Patients were instructed to hold their breath in normal expiration during the acquisition of the $\mathrm{CT}$ images. Positron emission tomography raw data were not used for readout. No contrast media was given during the PET/CT procedure.

\section{Imaging Assessment}

Three independent observers assessed the CT images ( 1 experienced radiologist, 1 experienced thoracic surgeon, and 1 experienced dual-board-certified nuclear medicine radiologist). The readers were blinded to any clinical information and unaware of pathologic staging or follow-up. The readers are further named CT-R1, CT-R2, and CT-R3. The PET/CT images were assessed by 3 independent observers ( 1 experienced radiologist and 2 experienced dual-board-certified nuclear medicine radiologists). The readers are further named PET/CT-R1, PET/CT-R2, and PET/CT-R3. Positron emission tomography/CT-R1 and PET/CTR3 were equal with CT-R1 and CT-R3. For the direct comparison between CT and PET/CT, only the results of PET/CT-R1 and PET/ CT-R3 were used. A commercially available PACS System (Impax 5; Agfa Healthcare, Dübendorf, Switzerland) was used for readout. All readers could use Multiplanar reformation tool and adjust window/level setting if necessary. Positron emission tomography/CT images were fused before reading using a commercially available workstation (Advantage Workstation, version 4.5; GE Healthcare).

TNM staging was performed using the TNM classification system for MPM based on the seventh edition of TNM classification of malignant tumors. ${ }^{17}$ For each patient, the precise TNM tumor stage was defined. Lymph nodes were considered as positive if the short-axis diameter was more than $1 \mathrm{~cm}$. Pathological TNM staging after EPP was used as reference.

\section{Statistical Analysis}

Relative and absolute underestimation or overestimation of clinical tumor stage compared with pathological tumor stage was calculated. Mean sensitivity, specificity, positive and negative predictive value, and accuracy of CT and PET/CT of all 3 readers were calculated (Analyse-it for Microsoft Excel, version 2.30;
Analyse-it Software, Ltd; http://analyse-it.com/; 2012). Underestimation was defined as false negative, and overestimation was defined as false positive. Interobserver agreement between the readers was tested using a weighted $\kappa$ test. $\kappa$ Values of 0.20 or less were considered as poor, $\kappa$ values of 0.21 to 0.40 as fair, $\kappa$ values 0.41 to 0.60 as moderate, $\kappa$ values of 0.61 to 0.80 as good, and $\kappa$ values of 0.81 to 1.00 as excellent agreement. ${ }^{18}$

\section{RESULTS}

\section{Patient Characteristics}

Digitally available chest CT scans performed after induction chemotherapy and before EPP were available from 62 mesothelioma patients (median age, 61 years [range, 38-72 years]; female: $n=9$; male: $n=53$ ). Induction chemotherapy consisted of 3 cycles of cisplatin and gemcitabine in 9 patients. Four years into the study, the induction regimen was changed, with the remaining 53 patients receiving cisplatin and pemetrexed. The majority of patients had an epithelioid histotype, $63 \%(n=39), 2 \%$ $(\mathrm{n}=1)$ sarcomatoid, and 35\% $(\mathrm{n}=22)$ biphasic histotype.

The distribution of the pathological $\mathrm{T}$ and $\mathrm{N}$ stages as well as IMIG stages among the population is displayed in Table 1.

\section{Computed Tomography}

The sensitivity, specificity, positive predictive value, negative predictive value, and accuracy of CT for stage T4 disease were $40 \%, 95 \%, 66 \%, 87 \%$, and $84 \%$ for CT-R1. Compared with pathological $\mathrm{T}$ staging, the number of correctly staged patients ranged from 40 (CT-R2) to 44 patients (CT-R1). The tumor extent was more often overestimated for stage T1 and T2 (CT-R1: $\mathrm{n}=2$ [3\%], CT-R2: $\mathrm{n}=3$ [4.5\%], CT-R3: $\mathrm{n}=4[6 \%])$ and underestimated for stages T3 and T4 (CT-R1 and CT-R3: $\mathrm{n}=14$ [22\%], CT-R2: $\mathrm{n}=17$ [27\%]) (Fig. 1, Table 2).

For nodal staging, the sensitivity, specificity, positive predictive value, negative predictive value, and accuracy for stage N2

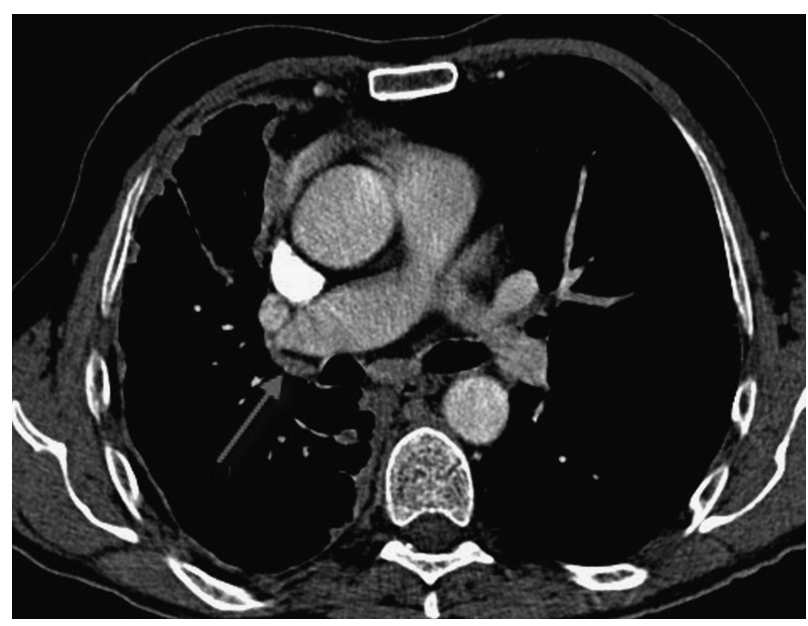

FIGURE 1. Computed tomography demonstrating tumor mass (arrow), staged as N0, with the patient stage N1 by pathology. 
TABLE 2. CT-Based Clinical Staging Compared With Pathological TNM Stages $(n=62)$

\begin{tabular}{lcccc}
\hline & & $\begin{array}{c}\text { Correct, } \\
\mathbf{n}(\%)\end{array}$ & $\begin{array}{c}\text { Underestimation, } \\
\mathbf{n}(\%)\end{array}$ & $\begin{array}{c}\text { Overestimation, } \\
\mathbf{n}(\%)\end{array}$ \\
\hline T stage & R1 & $44(72)$ & $14(22)$ & $4(6)$ \\
& R2 & $40(65)$ & $17(27)$ & $5(8)$ \\
N stage & R3 & $43(70)$ & $14(22)$ & $5(8)$ \\
& R1 & $49(79)$ & $7(11)$ & $6(10)$ \\
& R2 & $42(68)$ & $12(19)$ & $8(13)$ \\
IMIG & R3 & $47(76)$ & $9(15)$ & $6(10)$ \\
& R1 & $45(73)$ & $11(18)$ & $6(10)$ \\
& R2 & $41(66)$ & $16(26)$ & $5(8)$ \\
& R3 & $41(66)$ & $15(24)$ & $6(10)$ \\
\hline
\end{tabular}

and $\mathrm{N} 3$ disease were $70 \%, 97 \%, 85 \%, 88 \%$, and $87 \%$ (CT-R1). Lymph nodes were correctly staged between 42 (CT-R2) and 49 (CT-R1) patients. A large number of pathologic stage N0 were overestimated (CT-R1: $\mathrm{n}=4$, CT-R2: $\mathrm{n}=6$, CT-R3: $\mathrm{n}=4$ ). Four (CT-R1) and 7 patients (CT-R2, CT-R3) with pathological stage $\mathrm{N} 1 / \mathrm{N} 2$ were staged as N0.

Concerning the IMIG classification, the sensitivity, specificity, positive predictive value, negative predictive value, and accuracy of CT for IMIG stage IV disease were $50 \%, 89 \%$, $50 \%, 89 \%$, and $82 \%$ for CT-R1 (Fig. 2). The number of patients who were understaged was high for IMIG stages III and IV, with an underestimation of pathological stage III in 5 (CTR1), 10 (CT-R2), and 7 patients (CT-R3).

\section{$\mathrm{PET} / \mathrm{CT}$}

The sensitivity, specificity, positive predictive value, negative predictive value, and accuracy for stage T4 disease were $80 \%$, $95 \%, 80 \%, 95 \%$, and $92 \%$ for PET/CT-R2, which is higher when compared with CT. The number of correct T-stage patients was 20 (PET/CT-R2) and 21(PET/CT-R1/-R3). The tumor extent was more frequently underestimated than overestimated, but the percentage of underestimation was lower compared with CT (PET/ CT: $4 \%-19 \%$; CT: $22 \% \%-30 \%$ ), resulting in a higher negative predictive value and higher accuracy (Tables 3 and 4).

For nodal staging, the stage $\mathrm{N}$ sensitivity, specificity, positive predictive value, negative predictive value, and accuracy for stage
TABLE 3. PET/CT-Based Clinical Staging Compared With Pathological Stages $(n=26)$

\begin{tabular}{|c|c|c|c|c|}
\hline & Reader & $\begin{array}{c}\text { Correct, } \\
\text { n (\%) }\end{array}$ & $\begin{array}{c}\text { Underestimation, } \\
\text { n (\%) }\end{array}$ & $\begin{array}{c}\text { Overestimation, } \\
\text { n (\%) }\end{array}$ \\
\hline \multirow[t]{3}{*}{ T stage } & PET/CT-R1 & $21(81)$ & $4(15)$ & $1(4)$ \\
\hline & PET/CT-R2 & $20(77)$ & $5(19)$ & $1(4)$ \\
\hline & PET/CT-R3 & $21(81)$ & $1(4)$ & $4(15)$ \\
\hline \multirow[t]{3}{*}{ N stage } & PET/CT-R1 & $13(50)$ & $6(23)$ & 7 (27) \\
\hline & PET/CT-R2 & $14(54)$ & $6(23)$ & $6(23)$ \\
\hline & PET/CT-R3 & $15(58)$ & 7 (27) & $4(15)$ \\
\hline \multirow[t]{3}{*}{ IMIG } & PET/CT-R1 & $21(81)$ & $4(15)$ & $1(4)$ \\
\hline & PET/CT-R2 & $21(81)$ & $4(15)$ & $1(4)$ \\
\hline & PET/CT-R3 & $21(81)$ & $2(12)$ & $3(12)$ \\
\hline
\end{tabular}

N2 and N3 disease were $80 \%, 78 \%, 57 \%, 91 \%$, and $78 \%$ for PET/ CT-R2, which is lower compared with CT. N stage was correctly assessed in $54 \%$ of cases. Concerning underestimation or overestimation, no trend was evident (Fig. 3).

The sensitivity, specificity, positive predictive value, negative predictive value, and accuracy of CT for IMIG stage IV disease were $66 \%, 95 \%, 66 \%, 95 \%$, and $91 \%$ for PET/CT-R2 (Fig. 4). The interobserver agreement was good for all 3 readers (PET/ CT-R1 to PET/CT-R2: 0.82; PET/CT-R1 to PET/CT-R3: 0.78; PET/CT-R2 to PET/CT-R3: 0.79).

\section{Comparison Between CT and PET/CT for Patients Undergoing Both Examinations}

Comparing imaging to pathology for each patient individually, $\mathrm{T}$ and $\mathrm{N}$ staging was identical in 4 patients on all modalities. When focusing on IMIG classification, this number increased to 13 patients out of 26 , whereas a higher number of cases were overstaged by CT when compared with PET/CT. N stage was more often understaged on PET/CT compared with CT; especially pathologic N stage 2 was staged as N0 on PET/CT (Fig. 5).

\section{Interobserver Agreement}

For CT-based $\mathrm{T}$ staging and $\mathrm{N}$ staging, the interobserver agreement was moderate to good (CT-R1 to CT-R2: 0.73; CT-R1 to CT-R3: 0.52 ; CT-R2 to CT-R3: 0.48 ) and fair to moderate

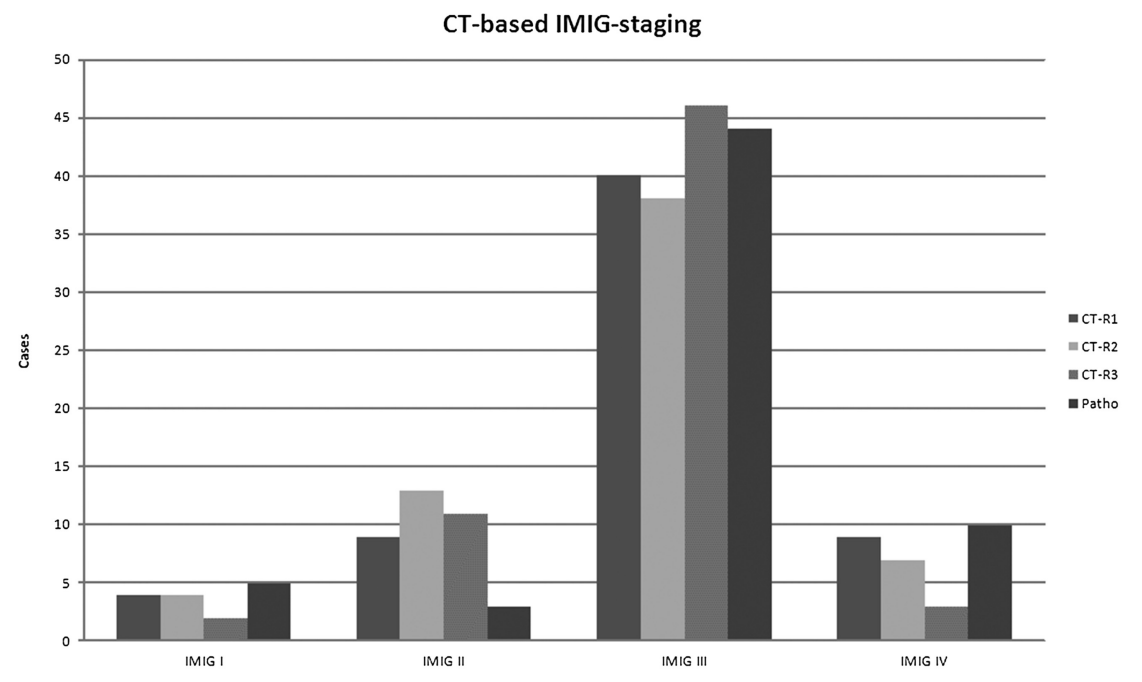

FIGURE 2. Computed tomography-based clinical IMIG classification for the 3 readers in comparison to pathological IMIG stage. 
TABLE 4. CT- and PET/CT-Based Staging Compared With Pathology for Each Patient (Population $n=26$ )

\begin{tabular}{lccc}
\hline & \multicolumn{1}{c}{ T stage } & N stage & IMIG \\
\hline CT and PET/CT correct & R1: $\mathrm{n}=17, \mathrm{R} 2: \mathrm{n}=18$ & $\mathrm{R} 1: \mathrm{n}=12, \mathrm{R} 2: \mathrm{n}=13$ & $\mathrm{R} 1: \mathrm{n}=15, \mathrm{R} 2: \mathrm{n}=17$ \\
CT and PET/CT understaged & $\mathrm{R} 1: \mathrm{n}=1, \mathrm{R} 2: \mathrm{n}=1$ & $\mathrm{R} 1: \mathrm{n}=3, \mathrm{R} 2: \mathrm{n}=4$ & $\mathrm{R} 1: \mathrm{n}=0, \mathrm{R} 2: \mathrm{n}=0$ \\
CT and PET/CT overstaged & $\mathrm{R} 1: \mathrm{n}=0, \mathrm{R} 2: \mathrm{n}=2$ & $\mathrm{R} 1: \mathrm{n}=4, \mathrm{R} 2: \mathrm{n}=3$ & $\mathrm{R} 1: \mathrm{n}=0, \mathrm{R} 2: \mathrm{n}=0$ \\
CT correct, PET/CT understaged & $\mathrm{R} 1: \mathrm{n}=3, \mathrm{R} 2: \mathrm{n}=0$ & $\mathrm{R} 1: \mathrm{n}=3, \mathrm{R} 2: \mathrm{n}=3$ & $\mathrm{R} 1: \mathrm{n}=4, \mathrm{R} 2: \mathrm{n}=4$ \\
CT correct, PET/CT overstaged & $\mathrm{R} 1: \mathrm{n}=1, \mathrm{R} 2: \mathrm{n}=2$ & $\mathrm{R} 1: \mathrm{n}=1, \mathrm{R} 2: \mathrm{n}=1$ & $\mathrm{R} 1: \mathrm{n}=0, \mathrm{R} 2: \mathrm{n}=1$ \\
CT understaged, PET/CT correct & $\mathrm{R} 1: \mathrm{n}=2, \mathrm{R} 2: \mathrm{n}=3$ & $\mathrm{R} 1: \mathrm{n}=2, \mathrm{R} 2: \mathrm{n}=1$ & $\mathrm{R} 1: \mathrm{n}=4, \mathrm{R} 2: \mathrm{n}=2$ \\
CT overstaged, PET/CT correct & $\mathrm{R} 1: \mathrm{n}=1, \mathrm{R} 2: \mathrm{n}=0$ & $\mathrm{R} 1: \mathrm{n}=1, \mathrm{R} 2: \mathrm{n}=1$ & $\mathrm{R} 1: \mathrm{n}=3, \mathrm{R} 2: \mathrm{n}=2$ \\
\hline
\end{tabular}

(CT-R1 to CT-R2: 0.43; CT-R1 to CT-R3: 0.51; CT-R2 to CT-R3: $0.37)$, respectively.

For PET/CT-based $\mathrm{T}$ staging and $\mathrm{N}$ staging, the interobserver agreement was good for both (T staging: PET/CT-R1 to PET/CTR2: 0.71; PET/CT-R1 to PET/CT-R3: 0.63; PET/CT-R2 to PET/ CT-R3: 0.64; N staging: PET/CT-R1 to PET/CT-R2: 0.75; PET/ CT-R1 to PET/CT-R3: 0.76; PET/CT-R2 to PET/CT-R3: 0.73).

\section{DISCUSSION}

Over the last decade, CT was commonly used for the initial evaluation of MPM, but it has limited sensitivity and specificity for the diagnosis and fails to predict resectability. ${ }^{7}$ Heelan et $\mathrm{al}^{5}$ compared CT and MRI for staging for MPM and found an accuracy between $55 \%$ for invasion of diaphragm and $80 \%$ for mediastinal fat invasion to correspond with stage T3. With the introduction of new types of CT scanners with higher temporal and spatial resolution as well as the availability of PET/CT scanning, it has to be assumed that the accuracy for staging the intrathoracic extent of MPM should have increased.

In our study, PET/CT and CT underestimated the local tumor extent in up to $30 \%$ of the cases. Sensitivity and accuracy for nodal staging were almost equal for CT compared with PET/CT, although it was often underestimated. Comparing PET/CT to CT alone, PET/CT improved the accuracy of preoperative IMIG staging, although the accuracy for $\mathrm{N}$ staging remained low for PET/CT. The higher accuracy for IMIG stage IV classification with PET/CT compared with CT is mostly based on the combination of $\mathrm{T}$ and $\mathrm{N}$ stage among the classes. The IMIG classification accommodates the low accuracy of lymph node staging by combining $\mathrm{N} 1$ and $\mathrm{N} 2$ into 1 stage (stage III). This raises the question of the appropriateness of the $\mathrm{T}$ and $\mathrm{N}$ descriptors for early-stage disease, which has also been discussed by Rusch et $\mathrm{al}^{6}$ after the initial analysis of the IASLC data set.

Computed tomography usually underestimated stage $\mathrm{T}$ disease by classifying actual stage T3 as stage T2, especially in regions of possible pericardial involvement, indicating that newer scanners still do not allow detection of invasion of the mediastinal fat accurately as the pleural layers are not visualized and the soft tissue contrast is reduced compared with MR. Alternatively, volumetry could replace the T descriptor. ${ }^{4,6,16}$

A high accuracy for PET/CT has been reported by other studies. ${ }^{1,3,7}$ Plathow et $\mathrm{al}^{3}$ evaluated and compared the accuracy of staging limited MPM between CT, PET/CT, and MRI. Their results showed a high accuracy for all 3 modalities for tumor IMIG stages II and III, whereas PET/CT was most accurate. Erasmus et $\mathrm{al}^{7}$ reported accuracies for the determination of stages $\mathrm{T}$ and $\mathrm{N}$ of $63 \%$ and $35 \%$ (study population $\mathrm{n}=29$ ). Stage $\mathrm{T}$ disease was more often understaged (29\%) as opposed to overstaged (7\%). The reason for understaging is the low spatial resolution of PET/CT not enabling the differentiation between T2 and T3 stages. Sørensen et $\mathrm{al}^{19}$ showed that PET/CT improved the accuracy of preoperative staging compared with $\mathrm{CT}$ alone. ${ }^{1}$ For the prediction of T4 disease, PET/CT had a sensitivity of $78 \%$ and specificity of $100 \%$.

Regarding nodal staging, the accuracy of PET/CT was lower compared with CT alone (78\%-87\%). We considered lymph node as positive if the short-axis diameter was greater than $1 \mathrm{~cm}$. Our results show that CT underestimated nodal stages N1 and N2 as reported by other studies. ${ }^{1,7}$ Pilling et $\mathrm{al}^{20}$ found an even lower sensitivity of $11 \%$ and accuracy of $66.6 \%{ }^{6}$ The reason therefore is 2-fold: (1) The close relation between primary tumor and hilar lymph nodes leads to misinterpretation of stage N1 as shown by other studies. ${ }^{1,3,7}$ (2) The use of $1 \mathrm{~cm}$ as cutoff is too high for this type of tumor, leading to underestimation. This is in line with the results of Nambu et al, ${ }^{21}$ who found a low sensitivity of CT for the presence of lymph node metastasis of non-small cell lung cancer using a cutoff of $5 \mathrm{~mm}$. On PET/CT, all N2 nodes that were not detected also showed no increased fluorodeoxyglucose uptake. The reason therefore might be the size of metastatic lymph nodes as shown for CT or microinvasion. The prognostic role of mediastinal lymph node involvement for MPM in general remains unclear. For mesothelioma, we and other groups feel that there might be rather direct invasion from adjacent tumor than lymphatic spread especially of the mammalian group of lymph nodes. ${ }^{6,22}$ This would not only lead to classifying this group of patients differently but also impact therapy decision making as many centers exclude patients with $\mathrm{N} 2$ disease for radical treatment. This theory is supported by the findings of Rusch et $a l,{ }^{6}$ as in the IASLC data set analysis, mediastinal lymph node involvement played no independent prognostic role.

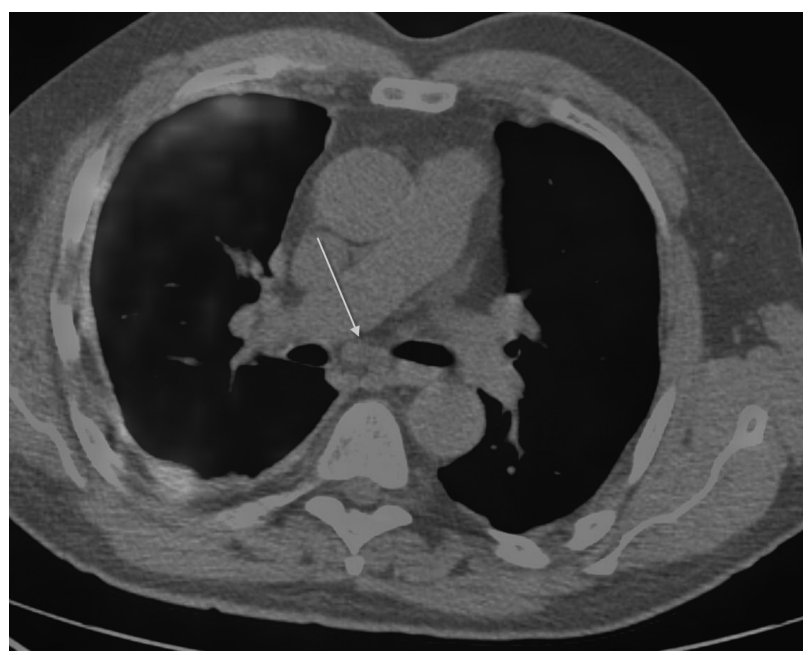

FIGURE 3. Patient with enlarged but FDG-negative lymph node on PET/CT. Lymph node was removed and pathologically staged as N2. 
PET/CT-based IMIG-classification

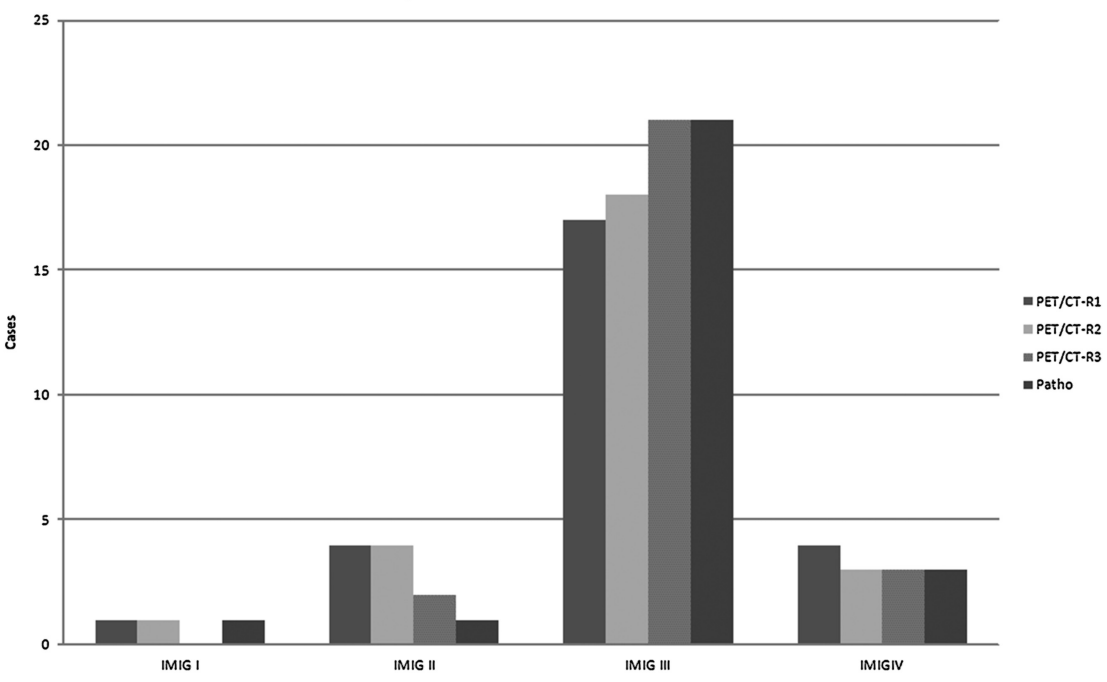

FIGURE 4. Positron emission tomography/CT-based clinical IMIG classification for all 3 readers in comparison to pathological IMIG stage.

Another imaging method to evaluate the intrathoracic tumor extent of MPM is MRI. In our study, we did not evaluate the accuracy or interobserver agreement of MRI assessing MPM. Nevertheless, several studies have shown that MRI describes the extent of tumor with high accuracy., ${ }^{3,23,24}$ Heelan et $\mathrm{al}^{5}$ showed that MRI is superior to CT for the evaluation of local invasion and the diaphragm. Plathow et $\mathrm{al}^{3}$ compared MRI to PET/CT and found that MRI is highly accurate in the local delineation of MPM and almost equal to PET/CT. The advantage of MRI is the high soft tissue contrast on unenhanced scans, in addition to the superior enhancement achieved postcontrast. Several studies have also shown that current sequences allow better delineation of the lesion and differentiation between pleural effusion, pleural thickening, and MPM compared with $\mathrm{CT}^{25-27}$

The interobserver agreement for local tumor extent was lower (moderate to good) for CT compared with PET/CT showing a "good" interobserver agreement. For nodal staging, the interobserver agreement was "fair to moderate" for CT and improved to "good" for PET/CT, although the accuracy was lower. The interobserver agreement for IMIG classification was "good" for all readers. There is only 1 other study assessing the interobserver agreement for IMIG classification of MPM. This study reported higher $\kappa$ values for IMIG classification compared with our study. ${ }^{3}$ The low interobserver agreement for $\mathrm{CT}$ and low accuracy reflect the limitations of CT to clearly differentiate among the stages. Although PET/CT has good interobserver agreement and good accuracy, it still leads to an underestimated clinical stage. The reason for the good interobserver agreement for IMIG is the setting of the classification. The combination of TNM stages for differentiating between IMIG stages II and III increases the robustness as mentioned before. The higher interobserver agreement in PET/CT favors this imaging modality.

Our study has several limitations. First, the number of patients undergoing PET/CT and $\mathrm{CT}$ in combination was low. In addition, a large number of stage T3 diseases are represented in this cohort (19 of 26 patients), which may bias our findings and results. Nevertheless, our population highlights the known weaknesses of the current imaging modalities. Second, we had different readers for CT and PET-CT. This was due to the lack of expertise for some readers in PET/CT. The third limitation is concerning the pathology as criterion standard. We are aware that the pathological samples from surgery are affected by preoperative imaging, and therefore the criterion standard is not truly independent. Nevertheless, it reflects the daily routine. Fourth, we included patients who underwent talc pleurodesis at the time of diagnostic biopsy. Although this causes inflammation
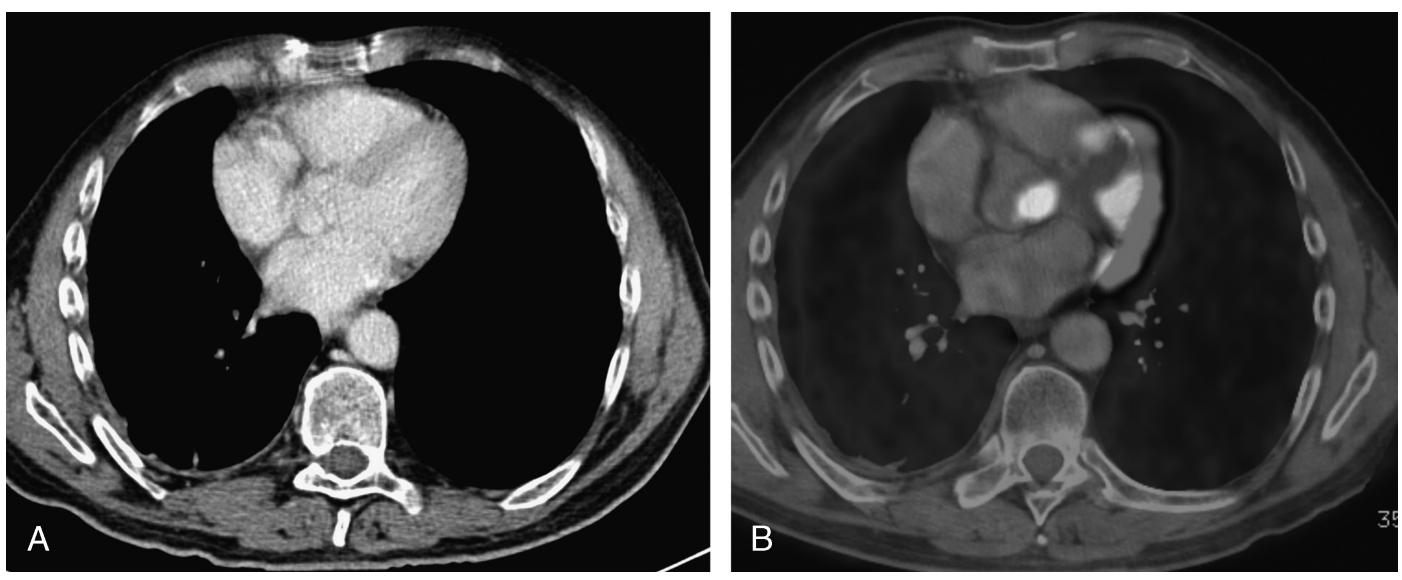

FIGURE 5. Patient with pathological stage T2. Computed tomography (A) and PET/CT (B) were assessed as stage $\mathrm{T} 1$ by all readers. 
that might influence the accuracy of PET/CT staging, Pilling et al found no significant difference regarding accuracy of intrathoracic staging. ${ }^{20}$

In conclusion, PET/CT underestimated the tumor stage in a substantial number of cases, making invasive staging methods such as mediastinoscopy still necessary. Regarding the local extent of the tumor to assess resectability, the inaccuracy of T and $\mathrm{N}$ staging of both methods shows that so far we do not have a reliable imaging technique, although PET/CT is clearly favored, particularly because of the lower interobserver variability for clinical staging of MPM compared with CT alone.

\section{REFERENCES}

1. Truong MT, Viswanathan C, Godoy MB, et al. Malignant pleural mesothelioma: role of CT, MRI, and PET/CT in staging evaluation and treatment considerations. Semin Roentgenol. 2013;48:323-334.

2. Yamamuro M, Gerbaudo VH, Gill RR, et al. Morphologic and functional imaging of malignant pleural mesothelioma. Eur J Radiol. 2007;64: 356-366.

3. Plathow C, Staab A, Schmaehl A, et al. Computed tomography, positron emission tomography, positron emission tomography/computed tomography, and magnetic resonance imaging for staging of limited pleural mesothelioma: initial results. Invest Radiol. 2008;43:737-744.

4. Armato SG 3rd, Labby ZE, Coolen J, et al. Imaging in pleural mesothelioma: a review of the 11th International Conference of the International Mesothelioma Interest Group. Lung Cancer. 2013;82: 190-196.

5. Heelan RT, Rusch VW, Begg CB, et al. Staging of malignant pleural mesothelioma: comparison of CT and MR imaging. AJR Am J Roentgenol. 1999;172:1039-1047.

6. Rusch VW, Giroux D, Kennedy C, et al. Initial analysis of the international association for the study of lung cancer mesothelioma database. J Thorac Oncol. 2012;7:1631-1639.

7. Erasmus JJ, Truong MT, Smythe WR, et al. Integrated computed tomography-positron emission tomography in patients with potentially resectable malignant pleural mesothelioma: staging implications. J Thorac Cardiovasc Surg. 2005;129:1364-1370.

8. American Joint Committee of Cancer. AJCC Cancer Staging Manual. 7th ed. New York, NY: Springer; 2010.

9. Cao CQ, Yan TD, Bannon PG, et al. A systematic review of extrapleural pneumonectomy for malignant pleural mesothelioma. J Thorac Oncol. 2010;5:1692-1703.

10. Kindler HL. Robust data: the essential foundation of a revised staging system for pleural mesothelioma. $J$ Thorac Oncol. 2012;7:1623-1624.

11. Lang-Lazdunski L, Bille A, Lal R, et al. Pleurectomy/decortication is superior to extrapleural pneumonectomy in the multimodality management of patients with malignant pleural mesothelioma. $J$ Thorac Oncol. 2012; 7:737-743.

12. Sugarbaker DJ, Strauss GM, Lynch TJ, et al. Node status has prognostic significance in the multimodality therapy of diffuse, malignant mesothelioma. J Clin Oncol. 1993;11:1172-1178.
13. Van Schil P. Malignant pleural mesothelioma: staging systems. Lung Cancer. 2005;49(suppl 1): S45-S48.

14. Butchart E, Ashcroft T, Barnsley W, et al. Pleuropneumonectomy in the management of diffuse malignant mesothelioma of the pleura. Experience with 29 patients. Thorax. 1976;31:15-24.

15. Rusch VW. A proposed new international TNM staging system for malignant pleural mesothelioma. From the International Mesothelioma Interest Group. Chest. 1995;108:1122-1128.

16. Frauenfelder T, Tutic M, Weder W, et al. Volumetry: an alternative to assess therapy response for malignant pleural mesothelioma? Eur Respir J. 2011;38:162-168.

17. International Union Against Cancer. TNM Classification of Malignant Tumours. 7th ed. Oxford, UK: Wiley-Blackwell; 2009.

18. Landis JR, Koch GG. An application of hierarchical kappa-type statistics in the assessment of majority agreement among multiple observers. Biometrics. 1977;33:363-374.

19. Sørensen JB, Ravn J, Loft A, et al. Preoperative staging of mesothelioma by 18F-fluoro-2-deoxy-D-glucose positron emission tomography/computed tomography fused imaging and mediastinoscopy compared to pathological findings after extrapleural pneumonectomy. Eur J Cardiothorac Surg. 2008;34:1090-1096.

20. Pilling J, Dartnell JA, Lang-Lazdunski L. Integrated positron emission tomography-computed tomography does not accurately stage intrathoracic disease of patients undergoing trimodality therapy for malignant pleural mesothelioma. Thorac Cardiovasc Surg. 2010;58:215-219.

21. Nambu A, Kato S, Motosugi U, et al. Thin-section CT of the mediastinum in preoperative $\mathrm{N}$-staging of non-small cell lung cancer: comparison with FDG PET. Eur J Radiol. 2010;73:510-517.

22. Zucali PA, Giovannetti E, Destro A, et al. Thymidylate synthase and excision repair cross-complementing group-1 as predictors of responsiveness in mesothelioma patients treated with pemetrexed/carboplatin. Clin Cancer Res. 2011;17:2581-2590.

23. Zahid I, Sharif S, Routledge T, et al. What is the best way to diagnose and stage malignant pleural mesothelioma?. Interact Cardiovasc Thorac Surg. 2011;12:254-259.

24. Toloza EM, Harpole L, McCrory DC. Noninvasive staging of non-small cell lung cancer: a review of the current evidence. Chest. 2003;123: $137 \mathrm{~S}-146 \mathrm{~S}$.

25. Ahamad A, Stevens CW, Smythe WR, et al. Promising early local control of malignant pleural mesothelioma following postoperative intensity modulated radiotherapy (IMRT) to the chest. Cancer J. 2003;9: 476-484.

26. Both M, Schultze J, Reuter M, et al. Fast T1- and T2-weighted pulmonary MR-imaging in patients with bronchial carcinoma. Eur J Radiol. 2005;53:478-488.

27. Flores RM, Akhurst T, Gonen M, et al. Positron emission tomography defines metastatic disease but not locoregional disease in patients with malignant pleural mesothelioma. J Thorac Cardiovasc Surg. 2003;126:11-16. 